\title{
Échotomographie : les nouveaux champs d'application
}

L'utilisation de l'échotomographie au cours d'endoscopies digestives ou d'interventions chirurgicales sur le rachis, le cerveau ou le foie, permet de localiser des lésions avec une grande précision. Quant au Doppler pulsé, il autorise l'exploration des vaisseaux profonds de façon non invasive.

Jean-Marc Lorphelin Chef de clinique-assistant Service de radiologie de l'hôpital Beaujon.

\section{ADRESSE ET TIRÉS A PART}

J. M. Lorphelin : Service de radiologie, hôpital Beaujon, roo, boulevard du Général-Leclerc, 92 I 8 Clichy Cedex.

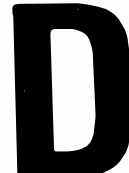

e récents progrès dans la technologie des sondes échographiques en temps réels ont été réalisés, comme la mise au point de sondes de haute définition permettant l'analyse des tissus superficiels avec une bonne résolution, la miniaturisation des sondes, et le couplage au niveau de la même tête de l'échotomographe temps réel et du Doppler pulsé, autorisant ainsi une analyse simultanée. Ces progrès techniques ont élargi le champ d'application de l'échotomographie aux examens per-opératoires et à l'endoscopie digestive. Le Doppler pulsé permet l'analyse des flux dans les vaisseaux abdomino-pelviens profonds.

\section{L'échotomographie per-opératoire}

Les applications de l'échotomographie per-opératoire (EPO) concernent surtout la neurochirurgie, ainsi que la chirurgie du foie et du pancréas. Elle permet une meilleure évaluation des lésions au cours de l'intervention et donc un geste plus précis ou mieux adapté.

En neurochirurgie : le système nerveux central chez l'adulte est inaccessible à l'échotomographie conventionnelle car le squelette crânio-rachidien absorbe complètement le faisceau ultrasonore. Une "fenêtre" acoustique est donc nécessaire : craniotomie ou laminectomie. L'examen se fait généralement avant l'ouverture des enveloppes méningées et peut contribuer à une limitation du champ opératoire.
Chez l'enfant, l'examen peut se faire par voie transfontanellaire.

Au niveau de l'encéphale, l'EPO permet une évaluation rapide et dans tous les plans de l'espace, des lésions tumorales, infectieuses ou vasculaires. Leur volume, leur étendue, leur siège par rapport aux vaisseaux, aux scissures et aux structures ventriculaires sont ainsi précisés. L'EPO est précieuse pour la localisation des lésions profondes et de petite taille car elle donne une meilleure définition du champ opératoire, limitant ainsi les dommages sur les tissus sains superficiels.

L'EPO permet le guidage des ponctions biopsies, comme l'échotomographie conventionnelle. Après résection partielle, l'EPO évalue le siège et la quantité de tissu pathologique résiduel. Certains auteurs [I] soulignent son intérêt dans l'évaluation des lésions tumorales à composante kystique (astrocytome) qui sont souvent mal analysées en tomodensitométrie : l'EPO favorise le repérage des cloisons dans les formations multiloculaires et de la composante solide intramurale. Puis elle permet de vérifier la qualité du drainage des cavités liquidiennes. L'EPO transfontanellaire est utilisée pour la mise en place des cathéters de drainage ventriculaire en cas d'hydrocéphalie [2]. La progression du drain est suivie de façon continue, ce qui peut éviter les complications classiques du cathétérisme "à l'aveugle" (hémorragie, encastrement dans les plexus choroïdes, mauvais positionnement).

En chirurgie de la moelle, outre 


\section{RÉFÉRENCES}

I. Rubin JM, Dohrmann GJ. Intraoperative neurosurgical ultrasound in the localization and characterization of intracranial masses. Radiology 1983; 148 : 519-24.

2. Merritt CR, Coulon $R$, Connolly E. Intraoperative neurosurgical ultrasound: transdural and transfontanelle applications. Radiology $1983 ; 148$ 513-7.

3. Rubin JM, Dohrmann GJ. The spinc and spinal cord during neurosurgical operations: realtime ultrasonography. Radiology 1985; $155: 197^{-}$ 2o

4. Plainfosse MC, Merran S. Intraoperative abdominal ultrasound. Radiology 1983; 147: 82932.

5. Belghiti J, Menu Y, Nahum H, Fekete F Apport de l'ćchotomographie per-općratoire dans la chirurgie des tumeurs du foie. Nouv Presse Med 1984; 13 : 1839-41.

6. Smith SJ, Vogelzang RL, Donovan J, Atlas SW, Gore RM, Neiman HL. Intraoperative sonography of the pancreas. $A 7 R$ 1985; $144: 557-62$.

7. Rifkin MD, Gordon SJ, Goldberg BB. Sonographic examination of the mediastinum and upper abdomen by fiberoptic gastroscope. Radiology 1984; 151 : 1 75-80.

8. Strohm WD, Kurtz W, Classen H. Les progrès diagnostiques de l'ultrasonographie endoscopique. Acta Endoscopica 1984; $14: 77-82$.

9. Rifkin MD, Kurtz AB, Choi HY, Golberg BB. Endoscopic ultrasonic evaluation of the prostate using a transrectal probe: prospective evaluation and acoustic characterization. Radiology I983; 149: 265-7 I

10. Fornage $\mathrm{BD}$, Touche $\mathrm{DH}$, Deglaire $\mathrm{M}$ Faroux MJ, Simatos A. Real-time ultrasoundguided prostatic biopsy using a new transrectal linear-array probe. Radiology $1983 ;$ 146: 547-8.

I1. Rifkin MD, Marks GJ. Transrectal US as an adjunct in the diagnosis of rectal and extrarectal tumors. Radiology 1985; $157: 499-502$.

12. Taylor KJ, Burns PN, Woodcock JP, Wells PNT. Blood flow in deep abdominal and pelvic vessels: ultrasonic pulsed Doppler analysis. Radiology 1985; 154: 487-93

13. Ohnishi K, Saito M, Koen H, Nakakama T, Nomura F, Okuda K. Pulsed Doppler flow as a criterion of portal venous velocity: comparison with cineangiographic measurements. Radiology I985; $154: 495^{-8}$.
Le Doppler pulsć permet de reconnaitre un vaisseau et d'analyser la circulation dans ce vaisseau. L'examen repose sur l'effet Doppler : des ultrasons émis avec une fréquence déterminéc (Fe) sont partiellement réfléchis par les globules rouges en mouvement avec une variation de fréquence que l'on appelle fréquence Doppler (Fd). Elle est proportionnelle à la vitesse $(\mathrm{V})$ de déplacement des globules rouges selon :

$$
\mathrm{Fd}=\frac{2 \mathrm{Fc} \times \mathrm{V}}{\mathrm{C}} \times \cos \theta
$$

C : représente la vitesse de propagation des ultrasons dans le milieu (environ I $500 \mathrm{~m} / \mathrm{s}$ ); $\theta$ : représente l'angle entre le faisceau incident et l'axe du vaisseau.

La sonde Doppler est à la fois émettrice et réceptrice. Elle émet de façon discontinue. Le vaisseau est d'abord repéré sur l'image échotomographique et la sonde Doppler étudie sélectivement les ultrasons réfléchis par les globules rouges de ce vaisseau. La vitesse de propagation des ultrasons étant presque identique dans tous les tissus, il suffit de mesurer le temps qu'ils mettent pour revenir, pour connaître la profondeur de la structure qui les réfléchit. Inversement, on peut sélectionner une profondeur et n' "écouter" que les échos qui reviennent dans le temps correspondant à cette profondeur. La mesure ne porte pas sur un point, mais sur un volume où les globules rouges n'ont pas tous la même vitesse de déplacement, sauf s'il s'agit d'un flux laminaire.

L'information contenue dans le signal Doppler peut être traduite sous la forme d'une courbe appclée analyse spectrale qui représente à chaque instant les différentes vitesses mesurćes avec leur énergic respective. Le sens de déplacement du flux peut également être déterminé.

La sonde Doppler et la sonde de repérage échotomographique sont ici séparées mais il existe aussi des sondes couplées. l'évaluation des lésions tumorales, l'EPO permet d'apprécier l'étendue des syringomyélies et le guidage de la pose d'un drain, ainsi que d'explorer des compressions de la moelle et des racines nerveuses créées par un processus tumoral ou par une hernie discale [3].

En chirurgie abdominale, plusieurs auteurs ont proposé d'utiliser l'EPO pour déterminer avec plus de précision le diagnostic et le siège des lésions tumorales ou lithiasiques hépatobiliaires, pancréatiques ou rénales. La lithiase biliaire [4] pose parfois des difficultés de diagnostic pré- ou per-opératoire (cholangiographie per-opératoire), en particulier s'il s'agit de microlithiase. L'EPO peut faire le diagnostic des petits calculs vésiculaires ou du bas cholédoque, mais ne se substitue pas aux méthodes d'investigations conventionnelles.

Pour les tumeurs du foie l'EPO améliore le pronostic opératoire [5] en facilitant la dissection chirurgicale par le repérage des vaisseaux et en permettant la mise en évidence de tumeurs non visibles ou non palpables. Les tumeurs primitives du foie (carcinomes hépatocellulaires) surviennent dans $90 \%$ des cas sur une cirrhose préexistante. La chirurgie comporte un double risque, per-opératoire et post-opératoire (hémorragie, insuffisance hépatique). Il est done indispensable de réaliser des résections aussi linitées que possible mais satisfaisantes sur le plan carcinologique. L'EPO paraît être la méthode la plus sensible d'évaluation de la diffusion et des rapports vasculaires des tumeurs primitives du foie. Dans les tumeurs secondaires (métastases de cancers coliques), l'EPO permet en outre de limiter l'étendue de la résection par un repérage précis des lésions (métastasectomies simples ou multiples, hépatectomie réglée). Dans les affections du pancréas, organe profond, l'exploration manuelle per-opératoire est le plus souvent facile. Néanmoins, l'EPO apporte des renseignements complémentaires intéressants dans l'exploration des lésions de pancréatite [6]. Elle permet d'apprécier la taille et l'aspect des canaux pancréatiques avant leur dissection. Elle est utile pour localiser et caractériser les 
pseudokystes, pour apprécier l'épaisseur de leur paroi et guider la ponction et le drainage. Tous ces renseignements ont une incidence sur la technique opératoire. Dans les cancers du pancréas, l'EPO présente moins d'intérêt. Elle permet cependant d'effectuer une biopsie orientée ou de découvrir des contreindications à la résection, qui étaient passées inaperçues avec les examens conventionnels pré-opératoires (thrombose vasculaire, extension hépatique).

Dans la lithiase rénale, l'EPO constitue une méthode sensible de repérage des calculs et intéressante pour apprécier l'extension à la veine rénale des cancers du rein.

\section{L'échotomographie endoscopique}

La présence d'air dans une structure anatomique rend son exploration impossible par l'échotomographie. L'œsophage et le médiastin sont masqués par l'air contenu dans les poumons; l'estomac et le duodénum contiennent généralement de l'air. La mise au point de petites sondes ultrasonores, incorporées à l'extrémité distale d'un fibroscope, permet l'exploration des parois digestives et des organes de voisinage, en complément de l'endoscopie. L'interprétation des images et l'orientation spatiale des structures examinées sont parfois difficiles et nécessitent une certaine habitude. L'image échotomographique des parois digestives est connue et comprend 4 couches. L'échotomographie endoscopique permet également l'exploration des organes. situés au contact du tube digestif : médiastin, lobe gauche du foie, vésicule, partie terminale de la voie biliaire principale, pancréas. Elle ne doit pas constituer un examen de routine, mais plutôt une technique d'exploration complémentaire pour les lésions mal documentées par les autres techniques d'imagerie ou par l'endoscopie [7].

Schématiquement, 3 types d'indication de l'échotomographie-endoscopique se dégagent [8] : bilan d'extension sousmuqueuse et médiastinale du cancer de l'œsophage et diagnostic des lésions sousmuqueuses de la paroi gastrique, explo- ration de la portion terminale du cholédoque (calculs, tumeurs papillaires), exploration du pancréas, en particulier pour ses portions céphalique et caudale. L'échotomographie endoscopique du pancréas a un bon pouvoir discriminatoire dans le diagnostic différentiel entre carcinome et pancréatite.

L'échotomographie transrectale est d'utilisation plus ancienne. Elle ne nécessite pas de fibroscope, une sonde adaptée à cette voie d'abord est utilisée. Elle permet une évaluation plus sensible qu'avec l'échotomographie suspubienne conventionnelle des petites lésions focalisées de la prostate [9]. Elle améliore la discrimination entre lésions bénignes et malignes et permet de guider la biopsie prostatique [10]. Plus récemment, deux auteurs ont proposé l'utilisation de l'échotomographie intrarectale pour le bilan d'extension des cancers rectaux [II]. Les résultats initiaux, comparables à ceux de la tomodensitométrie, demandent à être confirmés par une étude plus large.

\section{Abdomen et Doppler pulsé}

Le Doppler pulsé favorise l'étude d'une structure vasculaire, simultanément à l'échotomographie en temps réel. L'échotomographie permet le repérage du vaisseau et la définition du volume sanguin analysée par l'effet Doppler. L'intérêt est la possibilité d'analyser le flux sanguin dans des structures vasculaires profondes, abdominales ou pelviennes, de façon sélective [12]. Chacune des branches abdominales de l'aorte est définie par un spectre Doppler spécifique que l'on peut comparer à une "signature ". La connaissance du flux vasculaire a un intérêt dans la pathologie de l'artère rénale au cours de l'hypertension artérielle ou dans l'évaluation d'un greffon rénal, dạns la pathologie de l'artère-mésentérique supérieure au cours de l'ischémie intestinale.

$\mathrm{Au}$ niveau du pelvis, l'analyse Doppler des vaisseaux à destinée utérine ou ovarienne améliore la connaissance de certaines maladies de l'ovaire, en particulier des stérilités. Le Doppler pulsé permet également l'étude des flux veineux, en particulier dans le système porte [13]. L'évaluation de l'hypertension portale, qu'elle s'accompagne ou non d'une thrombose, en est une application intéressante.

En conclusion, malgré l'apparition de nouvelles techniques d'imagerie, l'échotomographie a conservé ses indications. Son champ d'application s'est même étendu à d'autres structures anatomiques (thorax, système nerveux, tube digestif) grâce à son utilisation per-opératoire ou endoscopique. Enfin, son utilisation comme méthode de guidage a permis de pratiquer la ponction de lésions profondes de façon sélective ou d'analyser les flux au sein des vaisseaux abdomino-pelviens, autrefois inaccessibles par l'examen Doppler conventionnel

\section{Summary}

Intraoperative real-time ultrasonography has been shown recently to be a useful technique in neurosurgical operations of the spine and brain. It allows to evaluate the extent and precise locations of intramedullary or intracerebral lesions. The effectiveness of shunt placement in syringomyelia or hydrocephalia and the completeness of tumor removal can be determined. Intraoperative ultrasonography has also been proposed as a valuable aid in the surgical management of hepatic tumors, particularly in evaluating the spread of tumors to the portal or hepatic veins.

Endoscopic ultrasonography is a new complementary imaging modality which provides information on intramural lesions of the upper gastrointestinal tract that are not available from other imaging modalities. Transrectal sonography is used to evaluate rectal or prostatic disease and to guide biopsy. Ultrasonic pulsed-Doppler analysis of the deep-lying abdomino-pelvic vessels provides qualitative or quantitative information on presence or absence and direction of flow in a structure, particularly in celiac and mesenteric axis, in renal vessels and portal vein or portocaval anastomoses. 